\title{
Epidermoid Cyst Presenting in the Floor of the Mouth
}

\author{
Mohamed Hania $^{\mathrm{a}, \mathrm{c}}$, Christopher Mannion ${ }^{\mathrm{b}}$
}

\begin{abstract}
Dermoid cysts are developmental malformations due to a defect in the fusion of embryonic lateral mesenchymatic layers during the fifth week of embryological development. Thirty-four percent of dermoid cysts occur in the head and neck area, of which $6.5 \%$ are in the floor of mouth. We present a rare case of a 17-year-old female with epidermoid cyst presenting in the floor of the mouth. A working diagnosis of a "plunging ranula" was concluded following salivary gland multidisciplinary team (MDT) meeting and the patient subsequently underwent an excision of lesion with right sublingual gland via an intraoral approach under GA. However, the pathology revealed the lesion consistent with an epidermoid cyst. We discuss the numerous pathological conditions that can affect the floor of the mouth, and differential diagnoses of lesions of a development, inflammatory, obstructive or neoplastic origin. Imaging aids such as ultrasound, MRI, and CT of head with contrast area are of valuable importance for estimating tumor margins and lesion composition, and assessing infiltration in surrounding tissues along with MDT which give platform with other departments to help form a working diagnosis and guide surgical approach.
\end{abstract}

Keywords: Swelling; Intraoral swelling; Sporting injuries; Maxillofacial surgery; Excision biopsy; Soft tissue swelling; Dermoid cyst; Epidermoid cyst; Ranula

\section{Introduction}

Dermoid cysts are rare, slow-growing, benign, developmental cysts, and only $34 \%$ occur in the head and neck area, of which $6.5 \%$ are in the floor of mouth [1].

Dermoid cysts are developmental malformations caused by the inclusion of either ectoblastic, mesoblastic or endoblastic layer due to a defect in the fusion of embryonic lateral mesenchymatic layers during the fifth week of embryological development $[2,3]$.

\footnotetext{
Manuscript accepted for publication February 13, 2017

${ }^{a}$ OMFS Leeds General Infirmary, Leeds, UK

${ }^{b}$ Consultant and Clinical Associate Professor in Oral and Maxillofacial Surgery, Leeds Teaching Hospitals NHS Trust, Leeds, UK

${ }^{\mathrm{c} C}$ Corresponding Author: Mohamed Hania, OMFS Leeds General Infirmary, Leeds, UK. Email: mhania@tcd.ie
}

They are typically classified into three types according to their histological appearance: dermoid, epidermoid and teratoid. Dermoid and epidermoid cysts have quite similar histopathology characteristics in that they both keratinize epithelium with fluid filled lumens but differ in the fact that only dermoid cysts have dermal structures such as sebaceous gland, hair structures, etc. Teratoid cysts are varied, however, from the type of epithelium simple squamous to ciliated respiratory epithelium [4].

We present a rare case of epidermoid cyst presenting in the floor of the mouth.

\section{Case Report}

A 17-year-old female presented to oral medicine clinic following referral by GP with regard to swelling right submandibular region.

She advised she was not feeling fatigued, malaise or pyrexia. History of presenting swelling was first noticed in the past year but seemed to have grown slowly since. She felt the swelling intraorally (Fig. 1) and it was generally soft but tender to palpation and sometimes felt hard on swallowing. She denied any problems with speech, swallowing or airway when lying supine.

She was medically fit and well with co-codamol to manage to stress-related migraines. She never smoked and currently studied for A levels.

On examination, the swelling was visible extraorally (Figs. 2 and 3) with no cervical lymphadenopathy. Intraorally right side floor of the mouth appeared larger than left, which

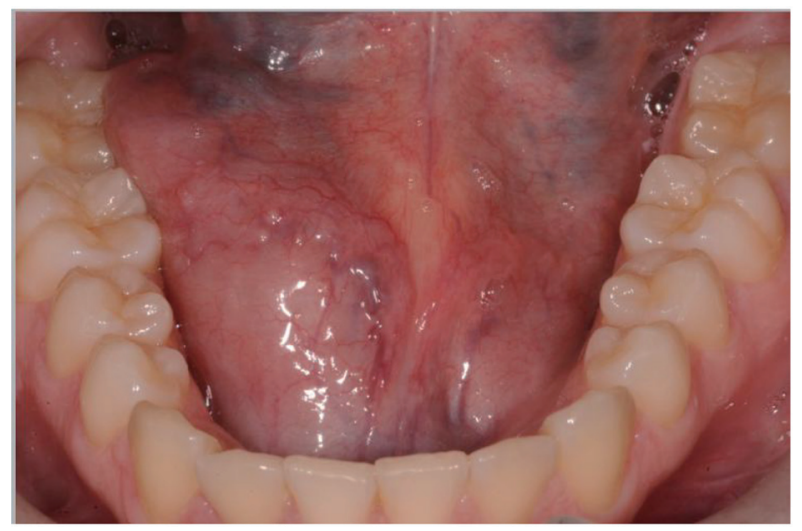

Figure 1. Intraoral photo of swelling right floor of the mouth. 


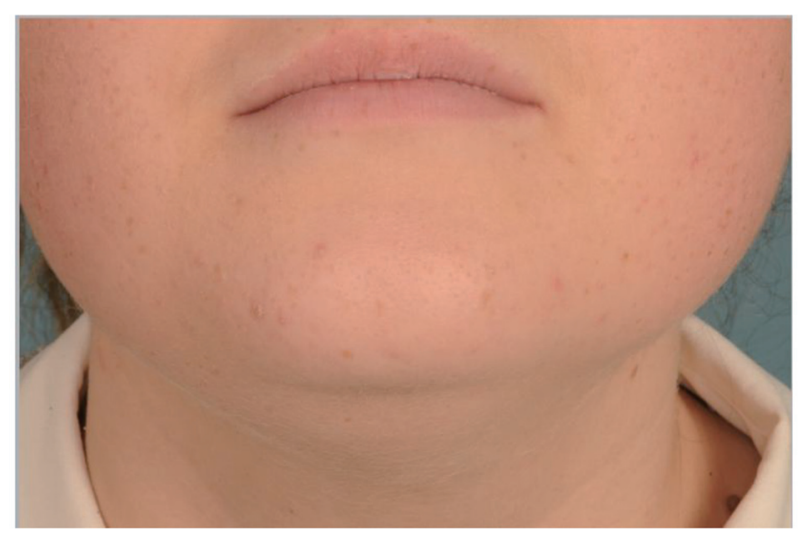

Figure 2. Extra oral photo of swelling (close up view).

was soft on bi-manual palpation. Saliva was readily expressed from Wharton's ducts. Linea Alba was recorded on right buccal mucosa.

Mandibular standard occlusal failed to show any radiopacity, so a plan of clinical photos and ultrasound was arranged. The ultrasound (Fig. 4) revealed an oval shaped lesion measuring $4.1 \times 3.3 \mathrm{~cm}$ separate from the mylohyoid muscle with echogenic material, no vascularity within, suggestive of dermoid/epidermoid cyst. An MRI (Fig. 5) was indicated at this point to characterize the lesion, which reported a $2.3 \times 3.7 \times$ 4.1 cyst on right sublingual region appearing to arise from the right sublingual gland.

The conflicting reports of the ultrasound and MRI coupled with reported increase in size of the swelling according to the patient led to her case being referred to a salivary gland multidisciplinary team (MDT) meeting. A working diagnosis of a "plunging ranula" was concluded due to very high T2 signal


Figure 3. Extra oral photo of swelling (further away).

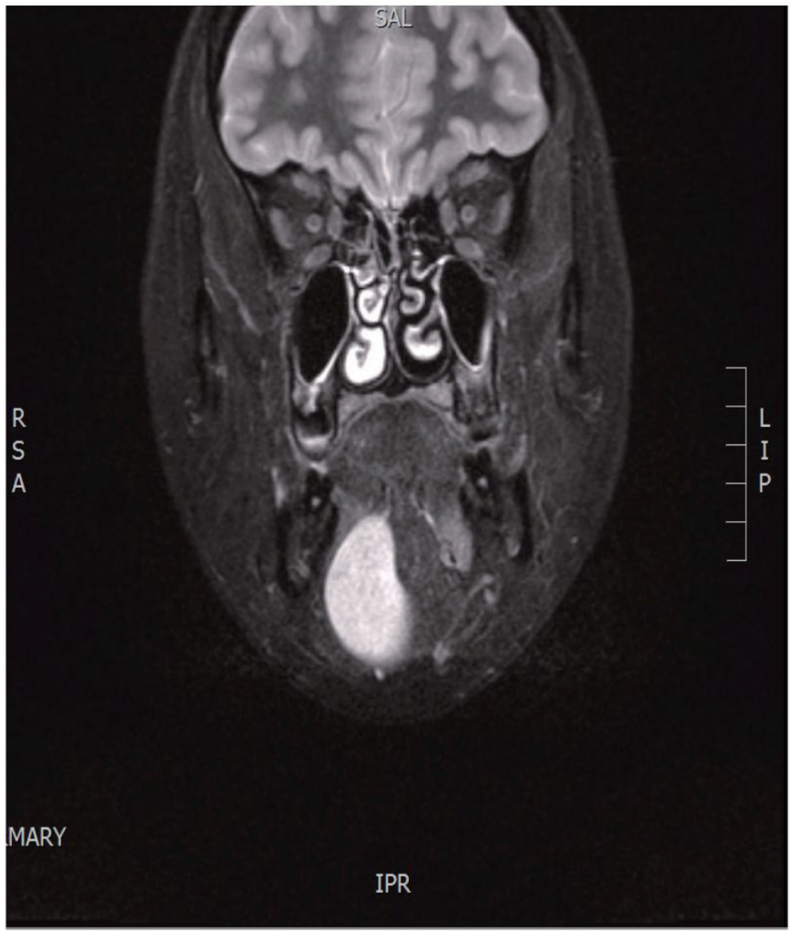

Figure 4. MRI scan showing the lesion.

indicating a high fluid content with proposed treatment of excision of lesion with right sublingual gland.

The patient along with family presented these findings and the patient subsequently underwent an excision of lesion with right sublingual gland via an intraoral approach under GA.

The patient made a full uneventful recovery . The pathol-

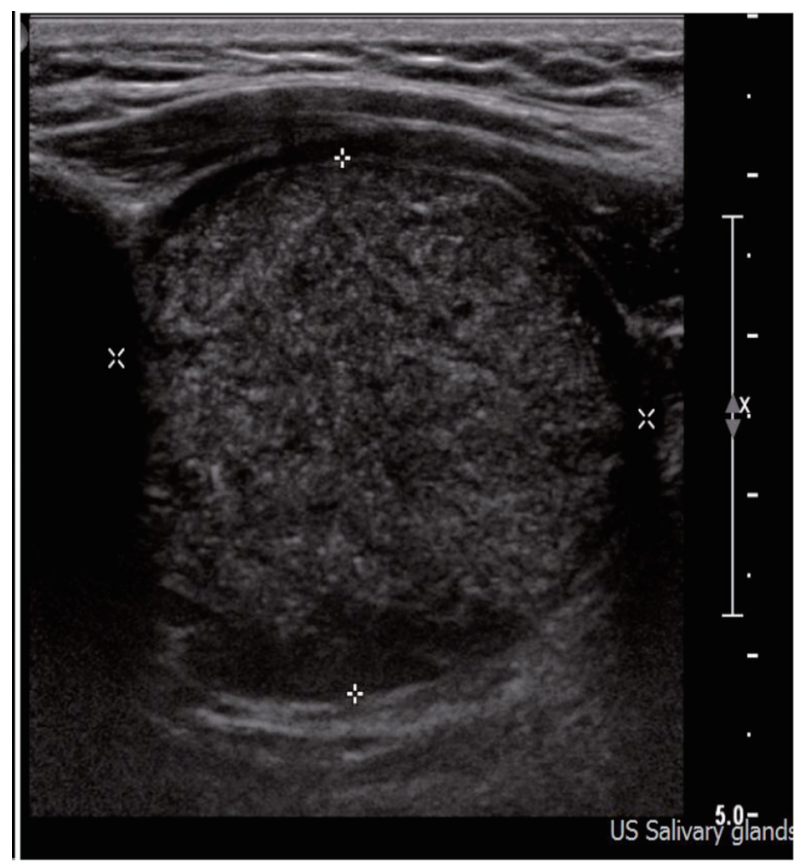

Figure 5. Ultrasound scan showing the lesion. 
ogy revealed normal morphology of right sublingual gland and the lesion as "fibrous cyst wall and a cyst lining composed of orthokeratinizing stratified squamous epithelium which has a corrugated surface appearance and keratin debris within the lumen", i.e. epidermoid cyst.

On postoperative follow-up, the intraoral area was observed to have healed quite nicely and patient reported no paraesthesia with good salivary gland function with no other symptoms.

\section{Discussion}

Dermoid cysts are rare, slow-growing, benign, developmental cysts and only $6.5 \%$ are in the floor of mouth [1].

While this was not the case in our patient, dermoid cysts may enlarge and interfere with deglutition and speech, or can pose a critical risk to the airway especially if there is an upward displacement of the tongue and therefore require immediate surgical intervention.

Depending on the position of development, i.e. above or below the mylohyoid muscle, sublingual or submandibular swelling can be caused. Surgical excision is the treatment of choice and recurrence is rare. An intraoral approach for the treatment of large lesions presenting above the mylohyoid muscle can provide good cosmetic and functional results.

However, adjunct diagnostic imaging aids such as ultrasound, MRI, and CT of head with contrast area are valuable tools for estimating tumor margins and lesion composition, and assessing infiltration in surrounding tissues.

MDT meeting provides helpful platform with surgical colleagues, radiologists and other departments to help form a working diagnosis. This mechanism should be utilized as it can also guide the surgical approach for each patient.

While the numerous pathological conditions can affect the floor of the mouth, the differential diagnosis must include lesions of a development, inflammatory, obstructive, or neoplastic in origin; however, other common pathologies of the oral soft tissue such as lipoma, branchial cleft cysts, and thyroglossal duct cysts should be ruled out [5].

A better understanding of epidermoid cyst, especially occurring intraorally, may add to the clinical care of patient with this rare disorder as it is not encountered often in practice.

\section{References}

1. Taylor BW, Erich JB, Dockerty MB. Dermoids of the head and neck. Minn Med. 1966;49(10):1535-1540.

2. Containment P. Medical-Encyclopedie chirurgicale-E20-860-A-10. Paris: Elsevier; 2000. Congenital fistulas and cysts of the neck.

3. Baliga M, Shenoy N, Poojary D, Mohan R, Naik R. Epidermoid cyst of the floor of the mouth. Natl J Maxillofac Surg. 2014;5(1):79-83.

4. Meyer I. Dermoid cysts (dermoids) of the floor of the mouth. Oral Surg Oral Med Oral Pathol. 1955;8(11):11491164.

5. Longo F, Maremonti P, Mangone GM, De Maria G, Califano L. Midline (dermoid) cysts of the floor of the mouth: report of 16 cases and review of surgical techniques. Plast Reconstr Surg. 2003;112(6):1560-1565. 